\title{
Automatic Recognition Algorithm of Information Architecture Reliability Based on Energy Internet Network Topology
}

\author{
Guowen Ren, ${ }^{1}$ Minrong Wu $\mathbb{D},{ }^{2}$ and Miao $\mathrm{Yu}^{3}$ \\ ${ }^{1}$ New Energy Research Institute, Chinese Society for Urban Studies, Beijing 100835, China \\ ${ }^{2}$ State Grid Ningxia Electric Power Co., Ltd., Ningxia 750001, China \\ ${ }^{3}$ Business School, China University of Political Science and Law, Beijing 102249, China
}

Correspondence should be addressed to Minrong Wu; 90102332@ncepu.edu.cn

Received 7 April 2021; Revised 5 July 2021; Accepted 20 July 2021; Published 26 August 2021

Academic Editor: Wenqing $\mathrm{Wu}$

Copyright (C) 2021 Guowen Ren et al. This is an open access article distributed under the Creative Commons Attribution License, which permits unrestricted use, distribution, and reproduction in any medium, provided the original work is properly cited.

\begin{abstract}
As a type of energy network, the scale of the power network is constantly expanding, and its structure has become more and more complicated. Correspondingly, the risks to the energy network are even less likely to be discovered, which will undoubtedly cause great troubles for the safe operation of the network. The traditional manual inspection method can no longer meet the requirements of huge and complex energy networks. Therefore, this paper proposes the algorithm, a research on the automatic identification of reliability of information architecture based on the topology of the energy Internet network. Abstract the server, storage, and other devices in the Energy Internet as network nodes, divide them according to service modules, and use Bayes estimation to evaluate and judge the impact of these nodes on the system function, so as to find the nodes with hidden risks. The results show that, compared with the traditional manual inspection method, the method proposed in this paper can efficiently and accurately find the nodes with risks and can help optimize the topology of the energy Internet network.
\end{abstract}

\section{Introduction}

Electric energy is one of the important energy sources for industrial development, and it is also the driving force for the sustainable development of social and economic development. The intelligent development of the power grid has also become the focus of research in various countries and regions [1]. However, with the increase in power demand, the power network has formed a huge interconnection network and has become more and more complex [2]. In the future, the power grid should provide safer, more reliable, clean, and highquality power sources to meet the various power needs of society. Due to the huge size and complexity of the energy internet, there are likely to be risks and hidden dangers, and the operational reliability of the energy internet is directly related to the social production and operation order $[3,4]$. If the energy internet fails, it is not only inefficient to rely on manual identification but it is also likely to be missed. Therefore, the research on the reliability identification of the information architecture of the energy internet will have a strong practical significance for the research and construction of the energy internet.

With the advancement of the smart grid, the addition of a large number of electronic devices and the introduction of new information technologies have brought many uncertainties and operational risks to the power network information system. In response to this problem, many experts and scholars have conducted research. In [5], the author pointed out that the traditional $10 \mathrm{kV}$ AC distribution network uses a large number of distributed energy and DC loads, which reduces the reliability of power supply and greatly increases the number of converters. In the study, the author proposed an AC/DC hybrid distribution network structure to ensure the reliability of the power supply. In [6], the author discusses potential network attacks and their adverse effects on power grid operation and proposes a general SCADA network attack process. In addition, the main challenges and strategies for protecting the smart grid from cyberattacks are also 
discussed. In [7], and the author proposed a social mobilization inspection system based on blockchain technology for external damage to the power grid, which helped to improve the detection level of power grid damage. In [8], according to the failure mechanism, failure type, and evolution characteristics of components in the relay protection system of intelligent substation, the author divides the factors that affect the operation status of equipment into long-term degradation failure and short-term impact failure. The single-configuration and dual-configuration risk assessment models of the relay protection system are established, and the degradation shock model is described based on the gamma distribution. In [9], the authors modeled and simulated the time-domain protection relays during the transmission of the power grid and used backup transient programs to simulate various failure scenarios. In [10], based on the hierarchical idea, the author used Markov chain and fault tree analysis to analyze the reliability of the wide-area measurement system and studied the importance of the wide-area communication components. In [11], the author proposes a high-voltage DC grid protection strategy to suppress DC fault current and prevent overcurrent in the branch of the modular multilevel converter. The strategy is based on the coordination of half-bridge modular multilevel converters and hybrid DC circuit breakers. In [12], in order to improve the reliability of the microgrid system, the author adopted a nonlinear signal transformation called "mathematical morphology" to introduce an intelligent differential protection scheme for the microgrid system. By considering various operating conditions such as microgrid topology (radial/grid) and microgrid operation mode (island/grid connection), many fault and no-fault conditions are simulated. In [13], the authors compared the performance of protection coordination (time setting and area selection interlock) and digital modular protection when deployed to gridconnected battery storage systems. The comparison between these protection management methods is based on their structure, function, and responsiveness to achieve a specific response. The above research has played a good role in protecting the power network. However, these methods have some shortcomings. First of all, substations, etc. tend to be networked, and the form of information sharing has brought a fundamental change to the relay protection structure model and also brought new problems to the reliability evaluation of relay protection. Second, the management of the huge power grid needs improvement, such as providing services through modular thinking, which can improve the efficiency of existing methods. Third, the reliability identification of the system is one of the important factors to ensure the smooth progress of the work. The reliability of existing research on large and complex systems is still insufficient, and there are many loopholes in the manual detection method of operation and maintenance personnel.

The energy network has the characteristics of large number and complicated structure. In the study of reliability identification, the topology of the energy internet network can be applied. The use of the characteristics of the network topology has yielded many good results. In [14], the author used the network topology for time series data measurement of the distribution network, basically projecting the actual voltage phasor pattern into the signal library related to the possible topological transition of a given distribution network. In [15], the author used the network topology to detect the essential proteins in the protein-protein interaction network, which effectively improved the prediction accuracy of essential proteins and was of great significance for understanding the cellular process. In [16], the author applied the network topology to key infrastructures such as the Internet and solved the problem that traditional packet-switched networks often suffer from long-term communication when network congestion occurs. In [17], the author applied the network topology to improve the computing network performance of the IHEP data center in China and improved the development efficiency, effectively responding to the problems of large storage resources and structural responsibility. In [18], the author integrated the network topology index into the study of the impact of the aquifer of the river characteristics. The results show that the network density index is a more powerful measurement standard and provides an improved accounting network topology in concept and function. This method has influence on the convection system. In [19], the author applied the network topology to the brains of patients with severe motor function and found that this method is helpful to help patients recover. It may take the role of compensation in the early stage of the disease, and the compensation gradually weakens later. When evaluating recovery, it is beneficial to consider changes in global and local parameters. In [20], the author applied network topology to the modeling of random processes at the boundary layer of complex systems to evaluate the service quality requirements of the NGN concept telecommunication network. In [21], the author uses the network topology for chaotic image encryption, so that multimedia information, images, etc. can be safely transmitted through unsecured channels such as social networks. In [22], the author applied the network topology to the study of food trade, and the insights obtained can be used to understand how to maintain fair access to resources in the world under climate change. In [23], the author's network topology is used for power network vulnerability analysis and provides the best attack strategy based on network congestion and maximum damage. Think of congestion as a cascading propagation mechanism and compare the reward functions based on increased congestion and immediate power outages. In [24], the author applied network topology to moving target tracking in a nonline-of-sight environment and extracted range estimates from received signal strength and time-of-arrival measurements to help a priori knowledge gained during target movement. In [25], the author used the network topology for the exploration of forming materials to rationalize the physical and mechanical properties. The above research shows that the network topology has great advantages in dealing with complex problems and can deal with problems with network structure. Therefore, it is considered to use the network topology to study the automatic identification algorithm of the reliability of energy information architecture.

This paper proposes an automatic recognition algorithm for the reliability of information architecture based on the 
energy Internet network topology. It solves the complexity and hugeness of the Energy Internet, and traditional manual inspection methods cannot meet the requirements of risk identification. This paper first abstracts devices such as servers, storage, and switches of the energy Internet as network nodes and abstracts the links between these devices to obtain network topology information. The obtained network topology is used to measure the distance, and an automatic reliability identification method is given. Through experiments, the method proposed in this paper can efficiently and accurately identify the risk nodes in the network, which provides practical reference for relevant maintenance personnel. In the experiment, the influence of noise on energy network performance evaluation was also considered. The Internet of energy continues to expand in scale, and the research in this paper provides a reference for the construction of related information systems.

\section{Automatic Recognition Method of Information Architecture Reliability Based on Energy Internet Network Topology}

\subsection{Energy Internet Network Topology Model}

2.1.1. Topological Composition of Energy Internet. Relying on the national backbone network, the Energy Internet connects several energy local area networks and other self-organized energy networks to realize on-demand energy allocation and intelligent management of power generation, transmission, distribution, and power supply. The energy local area network is composed of energy routers, distributed power generation equipment, energy storage equipment, and controllable load equipment. The energy local area network can work on-grid or off-grid to operate independently, that is, it is highly autonomous in the domain and interconnected between domains. The intelligent energy sensors in each energy local area network continuously collect various information such as power generation equipment, energy storage equipment, and loads in the energy local area network, and make energy control decisions based on the scheduling instructions of the upper-level transmission dispatching system. Then send control commands to the energy router for execution to achieve a high degree of integration of information flow and energy flow.

2.1.2. National and Regional Networks. First, construct a fully connected faction network with $N$ nodes to realize the interconnection of the national backbone network. This avoids the collapse of a large-scale energy network caused by a certain transmission line interruption under the deliberate attack of the energy internet and at the same time realizes the remote transmission of energy and on-demand scheduling. Each node here is a large-scale energy router, and the lower layer can be connected to the regional-level energy network as well as large-scale power generation equipment.

Second, the regional energy network serves the energy supply of the region internally and connects to other regions and backbone networks externally. Not only can it be directly connected to large and medium-sized power generation and storage facilities and directly provide energy for large enterprises and defense departments but also can realize energy transmission and distribution.

The basic model framework of the energy internet is as follows: (1) the interconnection of the backbone network improves the robustness of the energy internet and the freedom of energy scheduling at the global level. (2) The construction of regional hierarchical topologies facilitates efficient management of the energy internet and ensures critical load power supply and improves power supply reliability in the event of a serious power grid failure. (3) The "long side" within the hierarchical structure establishes and solves the special power needs of some regions, ensuring the normal operation of key facilities and important industries.

\subsection{Overall Plan for Automatic Risk Identification}

2.2.1. Basic Requirements for Safe and Stable Operation of Power Systems. In order to ensure the safe and stable operation of the power system, the basic requirements are as follows:

(a) In order to ensure the stability, frequency, and voltage level of the normal operation of the power system, the system should have sufficient static stability reserves, active and reactive power reserve capacity, and necessary adjustment means. At normal load fluctuations and when regulating active and reactive power flows, no sudden oscillations should occur

(b) The power grid structure is the basis for the safe and stable operation of the power system. In the planning and design, we should look at the overall situation and look forward to the overall consideration, rationally arrange the grid structure, strengthen the backbone network, and meet the following requirements: (1) it can adapt to the needs of development and changes and tide changes under various operating modes and has certain flexibility. (2) If any component is disconnected without failure, it should be able to maintain the stable operation of the power system and not cause other components to exceed the accident overload regulations. (3) It should have greater antidisturbance capability and meet the relevant requirements specified in this guideline. (4) Realize the principle of layering and zoning

(c) When the power system is damaged for any reason, predetermined measures must be taken to prevent the system from collapsing. Avoid long-term largescale power outages and catastrophic power outages for the most important users. The load loss should be minimized as much as possible, and the system should resume normal operation as soon as possible

2.2.2. Basic Idea of Automatic Reliability Identification Scheme. The composition of the energy information system includes multiple service modules, such as application-level service modules and storage-level service modules. Each service module of the information system needs to provide 
services to users or other constituent modules through a network link. At the same time, some service modules also usually need to access one or more other support service modules through a network link to provide external services. If the service node in the service module fails, other users or modules that need to access the service node will not be able to access the service node via the network. This also means that the network link from the user or other nodes to the abnormal node is not available. At this time, if the other nodes in the service module are in a normal operating state, and the user can access other normally operating nodes through other links, the work of the module will not be affected by a faulty node. Correspondingly, the network may fail to access the node because of a certain faulty node, then, this is a single point of failure.

In this paper, the service cluster is divided according to the functional characteristics of the energy network. After that, according to the topology structure of the energy internet network, the network link set through which all service cluster access relationships pass through is found. By calculating whether there is an available link in each link set when a single node device fails, it is determined whether the single node device is a single hidden point.

2.2.3. Reliability Assessment of the Module. The reliability of the module is the basis for evaluating the reliability of the system. The Bayesian estimation and correction method are used here. Unlike the general parameter estimation method, Bayes estimation does not assume that the parameter to be determined in the model formula is a fixed value constant, but instead sets it as a variable related to time $t$. Considering the randomness of the actual failure data, it is more scientific to set the parameter as a variable. Common parameter estimation methods only use overall information and sample information to estimate parameters. The concept of prior information was first proposed by Bayes. It combines the overall information of the failure data with the sample information of the real data to establish the concept of posterior information, obtains the prior distribution through the statistical characteristics of the failure data, and then speculates draw out the parameters in the model formula.

The prior distribution of the distribution function $F(x)$ of the population sample $X$ is $D P\left(\alpha, P_{0}\right), F_{0}(x)=P_{0}(X \leq x)$. Where $D P\left(\alpha, P_{0}\right)$ is called the Dirichlet distribution subject to the parameter $\alpha$, and $\alpha$ is a positive real number. If there is $p \sim \operatorname{Dir}(\alpha)$, the probability density function of $p=\left(p_{1}, \cdots\right.$, $\left.p_{m}\right)$ is needed, and the formula is as follows:

$$
\pi(p)=\frac{\Gamma\left(\sum_{j=1}^{m} \alpha_{j}\right)}{\prod_{j=1}^{m} \Gamma\left(\alpha_{j}\right)} p_{1}^{\alpha_{1}-1} \cdots p_{m}^{\alpha_{m}-1}, p_{j} \geq 0 .
$$

In the above formula, $p_{1}+p_{2}+\cdots+p_{m}=1$. The Bayesian estimation formula for the distribution function $F(x)$ of the total failure sample is as follows:

$$
\widehat{F}(x)=\sum_{i=1}^{h} \widehat{p}_{i}=\frac{\alpha}{\alpha+n} F_{0}(x)+\frac{1}{\alpha+n} \sum_{i=1}^{n} F_{0}\left(x \mid B_{i}\right) .
$$

\subsection{Reliability Identification Based on Energy Internet Network Topology}

2.3.1. Energy Network Topology Structure Ranging Method. Reasonable topology structure can realize state detection and reduce the probability of accidents, so as to quickly and accurately deal with and restore to normal state. At the same time, the distribution network is a kind of network with many nodes and complex structure. Due to daily maintenance, changes, construction, etc., there is a lack of realtime status monitoring and feedback in the distribution network. This also leads to possible errors in the actual network topology. In the network topology, the line length between two nodes can be measured by the signal transmission function of power line carrier communication, and the most important is the time of arrival (ToA) measurement. Serious errors will inevitably affect the reliability and stability of the power system. Therefore, the ToA problem is a parameter estimation of the cumulative exponential signal.

2.3.2. Reliability Identification Method. In this paper, the automatic identification of the reliability of the information architecture can be understood as the risk identification of the energy network. The main steps of this process are as follows: topology mapping and node initialization, definition of clusters, definition of access relationships between clusters, finding the set of access relationship links, and calculating the state value of nodes.

\section{(1) Topology mapping and node initialization}

Map the network topology diagram of the energy information system to the network connection diagram. In the network connection diagram, each node object contains three data attributes: node type, directly connected node set, and node status value. Among them, the node type storage area 0 or 1 is used to indicate whether the node belongs to a terminal node or a network node. A set of connected nodes stores a set of adjacent node objects directly connected to the node. The node status value storage area "+" or "-” is used to indicate whether the node status is normal or abnormal.

\section{(2) Definition of cluster}

Based on the architecture of the energy system, according to the principle of functional similarity, the nodes are divided into clusters. When a node is individually classified under a certain type of function, the single node is still regarded as a cluster. Number all clusters.

\section{(3) Definition of access relationship between clusters}

If there is an access relationship between the two clusters, $R$ is used to indicate this access relationship, and the access relationship will be numbered in order from 1 to $j$.

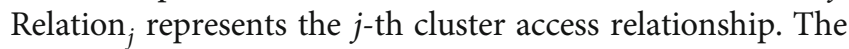

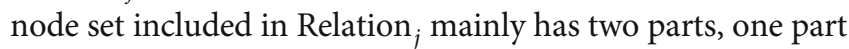
is the source node set. The other part is the collection of destination cluster nodes.

(4) Find out the collection of links to visit 


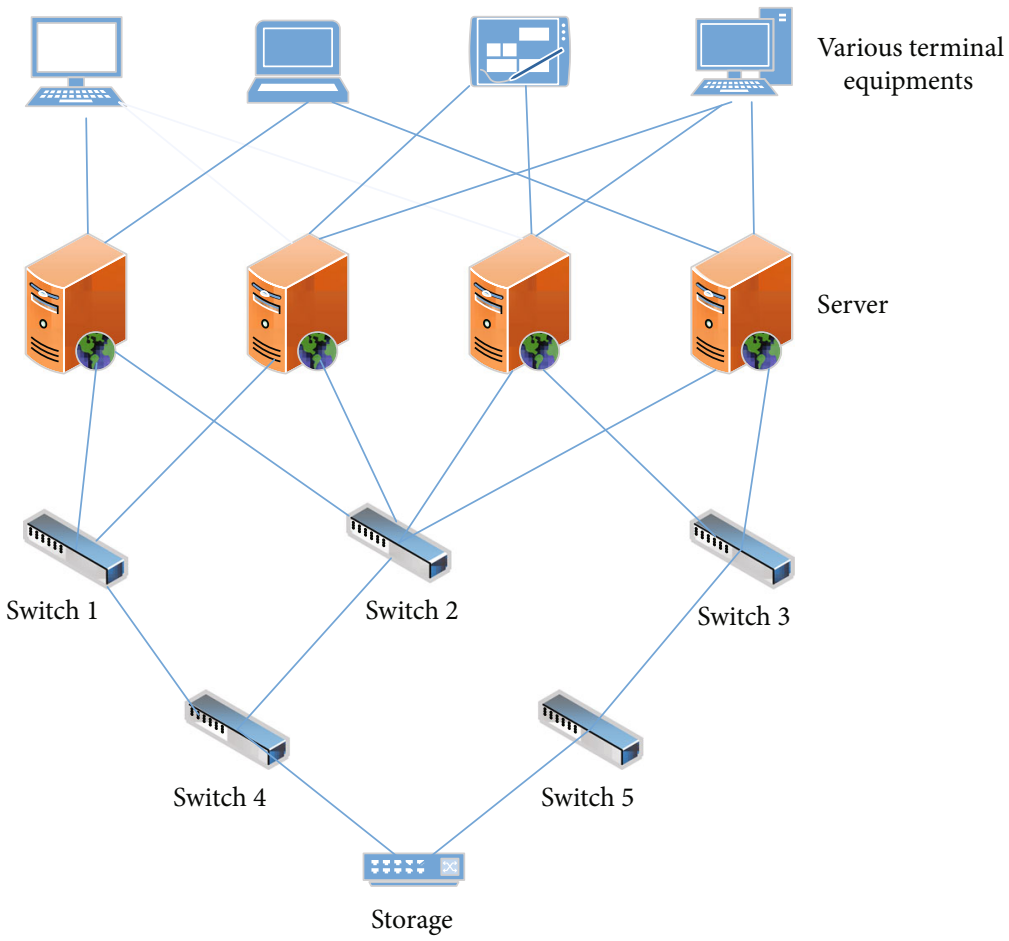

FIGURE 1: Energy network topology.

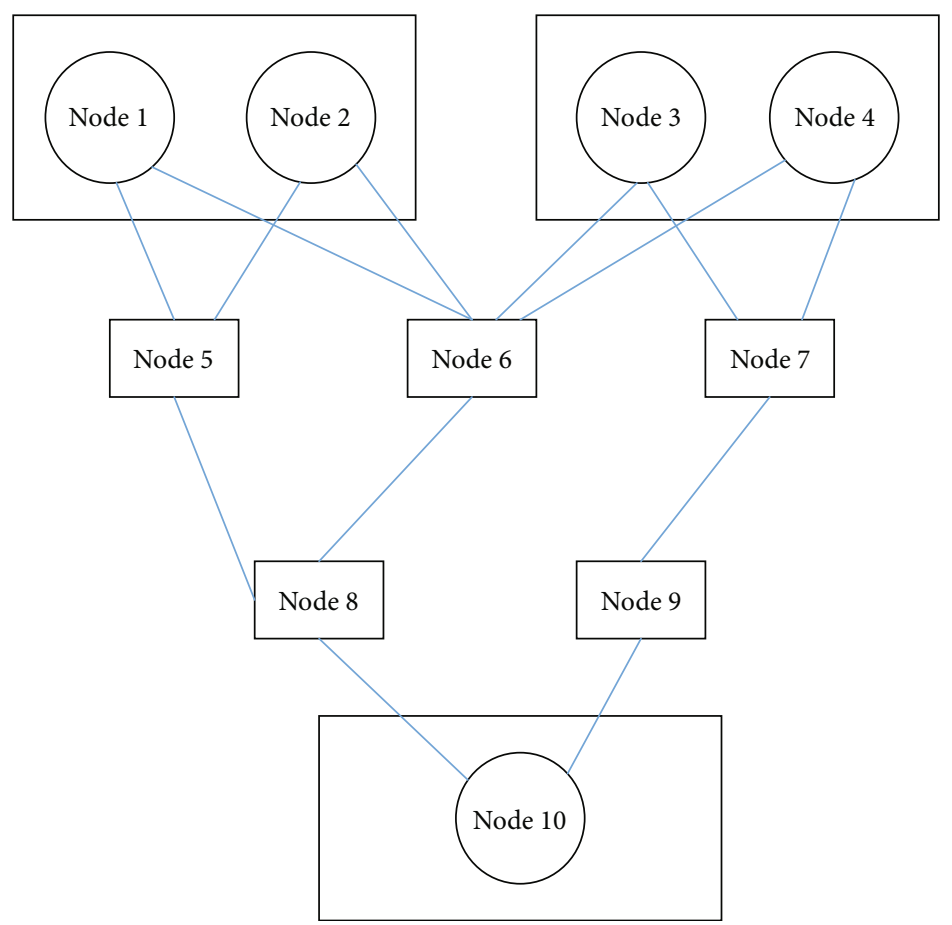

Figure 2: Topology map.

Since each node records neighbor node information, this information is similar to the next hop information in network routing. Therefore, it starts from a terminal node and transmits hop by hop through neighbor network nodes.
Finally, it will reach another terminal node to form a network link with the terminal node as the head/tail node and several network nodes as intermediate nodes. In this step, computer traversal calculations can be used to obtain network links 
from all end nodes to all other reachable end nodes. After calculating all network links, according to the terminal node information in the cluster access relationship, it is found that all cluster access relationships need to pass through the network link set. Let Relation $C_{j}$ denote the $j$-th cluster access relation network link set, and number the $k$ network links in the set from 1 to $k$ in sequence. That is, Link ${ }_{j-k}$ is used to denote the $k$-th network link in the $j$-th cluster access relationship.

(5) Calculate the state value of the node

Calculate the system function availability status value of each node in the system when a single node fails. To calculate the functional availability status value of each node, we first need to initialize and reset all node status values. Set the state value of the node to "-", and set the state value of the remaining $n-1$ nodes to " + ". Then calculate the state value of the link $\operatorname{Link}_{j-k}$, the state value of the network link set contained in the Relation $C_{j}$, and the availability state value in turn. Therefore, assuming that there are $n$ nodes in the energy internet, a total of $n$ calculations are required.

\section{Experiment of Automatic Recognition of Information Architecture Reliability}

This paper abstracts the power network and uses the network topology of the energy Internet information system to describe the interrelationships among various devices in the energy Internet. Forming the link between the devices, the devices of the energy Internet topology include devices such as switches, gateways, storage, and servers. The energy Internet topology is shown in Figure 1.

By using the energy Internet network topology structure information, the function availability status value of the information system network topology node is calculated to find hidden risks in the network, so as to help relevant personnel adjust the system architecture. One of the important methods for automatically identifying the reliability of information architecture is the distance measurement between nodes in the network. Here, in order to facilitate the identification and research of energy network topology, the signal transmission function of power line carrier communication is used for measurement. This method is expressed as the measurement of time of arrival.

\section{Results and Discussion of Automatic Identification of Information Architecture Reliability Based on Energy Internet Network Topology}

4.1. Function Availability State Value Calculation. According to the existing topology structure diagram of the Energy Internet, after removing various terminal devices in the network, the network topology diagram is mapped, and the mapping result is shown in Figure 2.
TABLE 1: State value results.

\begin{tabular}{lcccccccccc}
\hline Node & 1 & 2 & 3 & 4 & 5 & 6 & 7 & 8 & 9 & 10 \\
\hline Link set 1 & + & + & + & + & + & + & + & - & + & - \\
Link set 2 & + & + & + & + & + & + & + & + & + & - \\
Link set 3 & + & + & + & + & + & + & + & + & + & + \\
Functional availability & + & + & + & + & + & + & + & - & + & - \\
\hline
\end{tabular}

TABLE 2: Comparison of fit before and after.

\begin{tabular}{lccc}
\hline & Model 1 & Model 2 & Model 3 \\
\hline Original & 19.157 & 5.834 & 5.942 \\
Modified & 25.9 & 7.386 & 7.784 \\
Increase & $35.2 \%$ & $26.6 \%$ & $30.1 \%$ \\
\hline
\end{tabular}

TABLE 3: The obtained power channel model.

\begin{tabular}{lcccccc}
\hline Path number & 1 & 2 & 3 & 4 & 5 & 6 \\
\hline Length & 198 & 213 & 228 & 236 & 251 & 483 \\
Weight coefficient & 0.49 & 0.262 & -0.13 & 0.07 & -0.026 & -0.01 \\
\hline
\end{tabular}

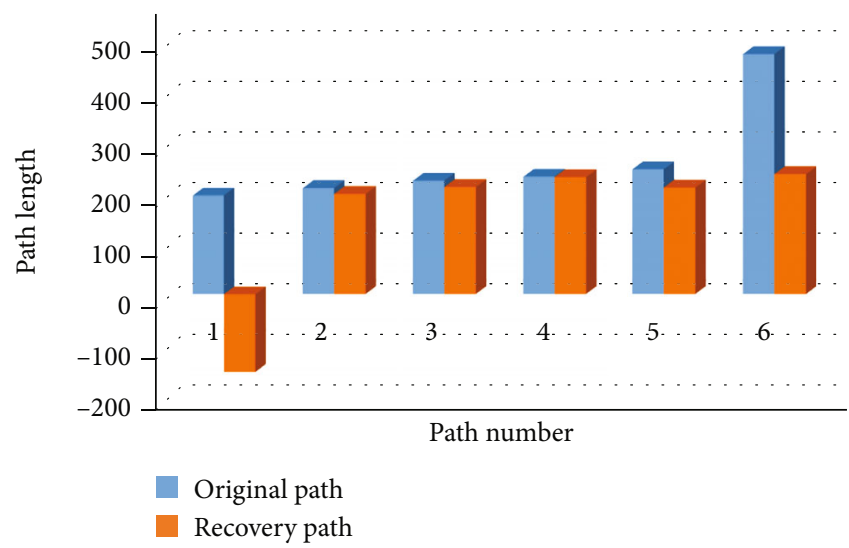

FIgURE 3: Channel estimation results when the signal-to-noise ratio is $60 \mathrm{~dB}$.

As shown in the figure above, there are 3 link sets. According to the reliability identification method proposed above, the access relationship link set is found, and the state value of the node is calculated. The final results are shown in Table 1.

As can be seen from Table 1, in the bottom column of functional availability, the representation form of node 8 and node 10 is "-", indicating that these two nodes are potential risk nodes. According to the analysis of the topology diagram, when node 8 fails, nodes 1 and 2 in the cluster cannot access storage node 10 , and the entire function of the cluster will be abnormal due to the inaccessibility of storage. Therefore, node 8 is indeed a hidden risk node, consistent with the calculation results. The storage nodes represented by node 10 are grouped into a cluster. Obviously, they are a single hidden risk node. In the process of calculating the availability of functions, node 8 and node 10 affect the access between clusters. Taking into account the reliability risks that exist here, 


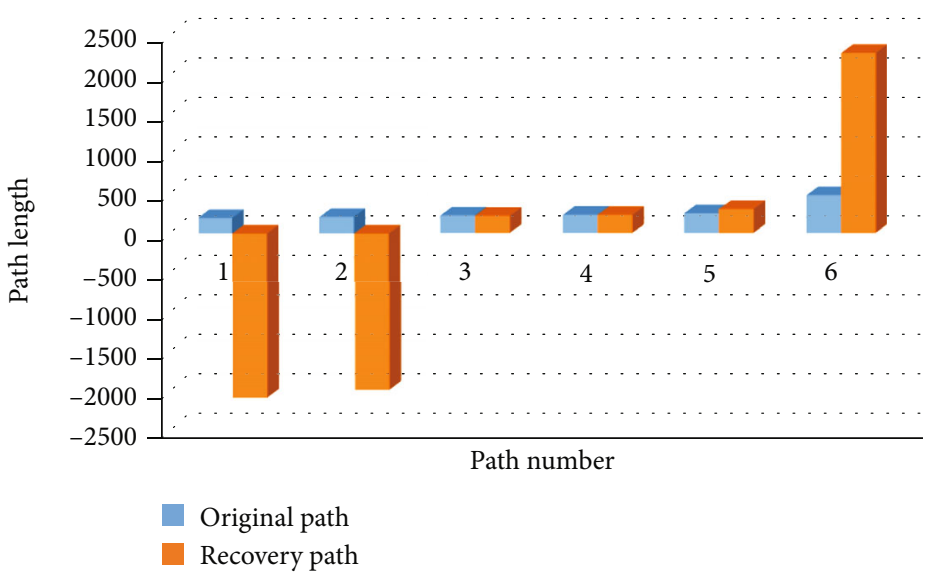

FIGURE 4: Channel estimation results when the signal-to-noise ratio is $30 \mathrm{~dB}$.

certain adjustments can be made, such as adjusting network connections and adding spare device nodes, to the dangers posed by the presence of a single node.

4.2. Validation of Bayesian Estimate Revision Validity. To date, the reliability assessment of information system architecture has not yet had a commonly used method. There are many factors that will affect the reliability of the module, and the same factor will affect the different modules differently. Therefore, the module reliability evaluation model with the highest fitting degree corresponding to each module is also different. First, verify that the Bayesian estimate is revised so that each model's ability to estimate and predict module failure data is improved. Compare the fits of the three different models before and after, as shown in Table 2.

It can be seen from Table 2 that the prediction ability of the module has been improved in different degrees after combining with the Bayesian estimation and correction, and the corresponding improvement of the fitting degree of the three is $35.2 \%, 26.6 \%$, and $30.1 \%$, respectively. The analysis found that before the correction, the difference was mainly due to the model mismatch caused by the difference in the statistical characteristics of the module data. The candidate model correction based on Bayes estimation effectively avoids the inaccurate model fitting caused by the deviation of the parameter estimation.

4.3. ToA-Based Ranging Performance Evaluation. In the experiment, a power line channel model obtained is shown in Table 3. This is a power line channel model with 6 diameters, and the length and strength of each diameter are indicated in the table. It can be seen from the channel model that between the two points of channel transmission, there are 6 channels from the direct path to the reflected path, and the shortest path has a maximum weight of 0.49 , which can be considered as a direct path. As the path becomes longer and the weight gradually decreases, other paths are complex reflection paths. The channel model will be used to estimate the time of arrival between two points. These reflection paths appear as multipaths that are continuously reflected between nodes other than the two points measured in the network. These numerical relationships are not directly controlled by ToA, but may be affected by the location of other nodes in the network. Therefore, in terms of measuring ToA between two points only, it is only necessary to measure the first diameter, which is the shortest straight diameter. However, in order to measure the overall topology of the network, ToA needs to be measured between any two points. Knowing the reflection path between every two nodes may be helpful for a more detailed and thorough understanding of the overall situation, so it is also very helpful to piggyback the value of the reflection path.

Add $60 \mathrm{~dB}$ and $30 \mathrm{~dB}$ noise to the channel and observe the recovery of each channel. The channel estimation results under different signal-to-noise ratios are shown in Figures 3 and 4.

It can be seen from Figures 3 and 4 that although the signal-to-noise ratio of $60 \mathrm{~dB}$ is already high, it still makes the estimation error. The presence of noise will affect the channel estimation results, mainly manifested in the emergence of negative value paths, as well as the misalignment and loss of paths. The accuracy of the method can be increased by discarding negative-value paths, but this reduces the total number of estimated paths, thereby reducing the sum of the number of reflected paths and the number of direct paths. The direct path is actually reserved by low errors, and high errors are mainly concentrated on the reflection path. In order to ensure the accuracy of measuring the direct path between two points, the accuracy of the reflection path is also required but not necessary, so it is necessary to verify the sensitivity of the direct path to noise. If the direct path is not sensitive to noise, the correct direct path can still be measured to provide correct information for the layer-bylayer reconstruction of the network. The inaccuracy of the information of the reflection path makes it necessary to discard them.

4.4. Comparison of Recognition Accuracy. In order to further explore the recognition accuracy level of the method proposed in this paper on the reliability identification of information architecture, here, we count the changes in the accuracy rate of the manual inspection method and the method proposed in this paper as the scale of the energy Internet continues to increase. As we all know, with the 


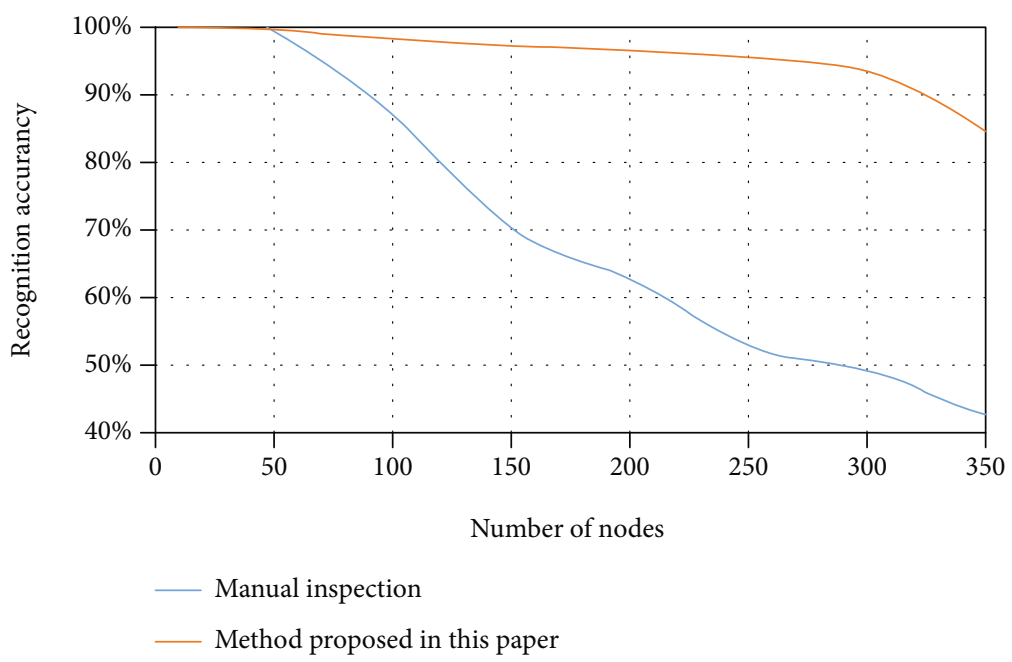

FIGURE 5: Change in recognition accuracy of different methods with network size.

increase of energy Internet nodes, the network has become more complex, and the determination of potential risks and faults on related nodes has become particularly difficult. The recognition rate of different methods on the expanding energy network is shown in Figure 5.

As can be seen from Figure 5, when the number of nodes is less than 50, the recognition accuracy of the proposed method and the manual inspection method is almost at the same level, and the difference is not very large. When the number of nodes in the network is higher than 50 , the difference between the two methods starts to become obvious. The inspection accuracy of the proposed method is much higher than that of the manual inspection method. However, when the number of nodes is higher than 300, the recognition rate of risk-risk nodes with more methods mentioned in this paper shows a significant downward trend. The analysis found that this is because as the number of network nodes continues to increase, the topology of the energy Internet becomes extremely complex, which exceeds the reasonable number of network nodes in the method designed in this paper, resulting in a decrease in accuracy. Therefore, in the follow-up research, improving the recognition accuracy of the method in the larger and more complex network is one of the key research directions.

\section{Conclusion}

The energy internet is constantly developing and growing, and its complexity and scale are changing accordingly. Based on the actual situation, this paper solves the problem of automatic identification of the reliability of related information architecture and proposes a research on the automatic identification of information architecture reliability based on the energy Internet network topology. This effectively solves the problem that the traditional manual method is seriously insufficient in inspection efficiency and accuracy. In the future, the energy internet in different countries and regions will evolve with different topological characteristics. The research in this article will provide a reference for practical problems in related fields.

\section{Data Availability}

This article does not cover data research. No data were used to support this study.

\section{Conflicts of Interest}

The authors declare that they have no conflicts of interest.

\section{Acknowledgments}

This study was funded by National Key R\&D Plan: 2021YFE0102400.

\section{References}

[1] I. Yildiz and H. Caliskan, "Energetic and exergetic carbon dioxide equivalents and prices of the energy sources for buildings in Turkey," Environmental Progress \& Sustainable Energy, vol. 37, no. 2, pp. 912-925, 2018.

[2] S. Z. Stefanov and P. P. Wang, "Taming the dragon-king of a day-ahead smart grid blackout," New Mathematics \& Natural Computation, vol. 14, no. 1, pp. 11-20, 2018.

[3] N. Ferraz Junior, A. Silva, A. Guelfi, and S. T. Kofuji, "Iot6sec: reliability model for internet of things security focused on anomalous measurements identification with energy analysis," Wireless Networks, vol. 25, no. 4, pp. 1533-1556, 2019.

[4] Z. Song, J. Zhang, X. Xiao, and D. Niu, "Multi-energy combined peak dispatching system synthetic benefit evaluation based on variable weight theory and matter-element extension model," International Journal of Energy Sector Management, vol. 13, no. 3, pp. 713-725, 2019.

[5] X. Xu, N. Tai, Y. Hu, W. Wang, F. Zheng, and W. He, "Reliability calculation of ac/dc hybrid distribution network with a solid-state transformer," The Journal of Engineering, vol. 2019, no. 16, pp. 3067-3071, 2019.

[6] L. A. Kumar, "Protecting power grid automation systems," Electrical India, vol. 59, no. 6, pp. 52-54, 2019, 56, 58-60.

[7] H. E. Bing, L. I. Ren-De, S. S. Wang, and L. Jian-Guo, “A social mobilized inspection system against external damage of power 
grid based on block chain technology," Journal of Physics Conference Series, vol. 1453, article 012104, 2020.

[8] F. Xiao, Z. Wang, Z. Zhang, X. Yin, and H. Li, "Study on maintenance strategy of relay protection system based on condition monitoring," Power System Protection \& Control, vol. 46, no. 6, pp. 74-83, 2018.

[9] J. P. G. Ribeiro and F. V. Lopes, "Modelling and simulation of a time-domain line protection relay," The Journal of Engineering, vol. 2018, no. 15, pp. 861-865, 2018.

[10] W. Zhang, J. Xu, Y. Li, and X. Zou, "Detecting essential proteins based on network topology, gene expression data, and gene ontology information," IEEE/ACM Transactions on Computational Biology and Bioinformatics, vol. 15, no. 1, pp. 109-116, 2018.

[11] S. Wang, C. Li, O. D. Adeuyi, G. Li, C. E. Ugalde-Loo, and J. Liang, "Coordination of mmcs with hybrid dc circuit breakers for hvdc grid protection," IEEE Transactions on Power Delivery, vol. 34, no. 1, pp. 11-22, 2019.

[12] M. Mishra, R. R. Panigrahi, and P. K. Rout, "A combined mathematical morphology and extreme learning machine techniques based approach to micro-grid protection," Ain Shams Engineering Journal, vol. 10, no. 2, pp. 307-318, 2019.

[13] S. A. Saleh, C. Richard, X. F. St Onge, J. Meng, and E. CastilloGuerra, "Comparing the performance of protection coordination and digital modular protection for grid-connected battery storage systems," IEEE Transactions on Industry Applications, vol. 55, no. 3, pp. 2440-2454, 2019.

[14] G. Cavraro and R. Arghandeh, "Power distribution network topology detection with time-series signature verification method," IEEE Transactions on Power Systems, vol. 33, no. 4, pp. 3500-3509, 2018.

[15] Z. H. Dai, Z. P. Wang, and Y. J. Jiao, "Reliability evaluation of the communication network in wide-area protection," IEEE Transactions on Power Delivery, vol. 26, no. 4, pp. 25232530, 2011.

[16] Y. Hu and M. Koibuchi, "Optimizing slot utilization and network topology for communication pattern on circuitswitched parallel computing systems," IEICE Transactions on Information and Systems, vol. E102.D, no. 2, pp. 247-260, 2019.

[17] A. Nechaevskiy, G. Ososkov, D. Pryahina, V. Trofimov, and W. Li, "Simulation approach for improving the computing network topology and performance of the China IHEP Data Center," The European Physical Journal Conferences, vol. 214, no. 5, article $08018,2019$.

[18] E. L. Heasley, N. J. Clifford, and J. D. A. Millington, "Integrating network topology metrics into studies of catchment-level effects on river characteristics," Hydrology and Earth System Sciences, vol. 23, no. 5, pp. 2305-2319, 2019.

[19] R. Chen, C. Li, and M. Su, "A study on the brain network topology attributes in stroke patients under severe motion function," Chinese Journal of Rehabilitation Medicine, vol. 34, no. 1, pp. 52-58, 2019.

[20] T. V. Azarnova, S. A. Barkalov, and V. V. Ukhlova, "Estimation of time characteristics of systems with network topology and stochastic processes of functioning," Journal of Physics Conference, vol. 1203, no. 1, article 012055, 2019.

[21] R. Vidhya and M. Brindha, "A novel conditional butterfly network topology based chaotic image encryption," Journal of Information Security and Applications, vol. 52, p. 102484, 2020.
[22] A. G. Dolfing, J. R. F. W. Leuven, and B. J. Dermody, "The effects of network topology, climate variability and shocks on the evolution and resilience of a food trade network," PLoS One, vol. 14, no. 3, article e0213378, 2019.

[23] C. Caro-Ruiz, A. S. al-Sumaiti, S. Rivera, and E. Mojica-Nava, "A mdp-based vulnerability analysis of power networks considering network topology and transmission capacity," IEEE Access, vol. 8, no. 1, pp. 2032-2041, 2020.

[24] S. Tomic, M. Beko, R. Dinis, and P. Montezuma, "Estimating directional data from network topology for improving tracking performance," Journal of Sensor and Actuator Networks, vol. 8, no. 2, p. 30, 2019.

[25] M. Formanek and F. Martelli, "Probing the network topology in network-forming materials: the case of water," $A I P$ Advances, vol. 10, no. 5, article 055205, 2020. 